\title{
Toward a Predictive Framework for Convergent Evolution: Integrating Natural History, Genetic Mechanisms, and Consequences for the Diversity of Life*
}

\author{
Anurag A. Agrawal ${ }^{\dagger}$ \\ Department of Ecology and Evolutionary Biology, Cornell University, Ithaca, New York 14853; and Department of Entomology, Cornell \\ University, Ithaca, New York 14853
}

\begin{abstract}
AвstraCt: A charm of biology as a scientific discipline is the diversity of life. Although this diversity can make laws of biology challenging to discover, several repeated patterns and general principles govern evolutionary diversification. Convergent evolution, the independent evolution of similar phenotypes, has been at the heart of one approach to understand generality in the evolutionary process. Yet understanding when and why organismal traits and strategies repeatedly evolve has been a central challenge. These issues were the focus of the American Society of Naturalists Vice Presidential Symposium in 2016 and are the subject of this collection of articles. Although naturalists have long made inferences about convergent evolution and its importance, there has been confusion in the interpretation of the pattern of convergence. Does convergence primarily indicate adaptation or constraint? How often should convergence be expected? Are there general principles that would allow us to predict where and when and by what mechanisms convergent evolution should occur? What role does natural history play in advancing our understanding of general evolutionary principles? In this introductory article, I address these questions, review several generalizations about convergent evolution that have emerged over the past 15 years, and present a framework for advancing the study and interpretation of convergence. Perhaps the most important emerging conclusion is that the genetic mechanisms of convergent evolution are phylogenetically conserved; that is, more closely related species tend to share the same genetic basis of traits, even when independently evolved. Finally, I highlight how the articles in this special issue further develop concepts, methodologies, and case studies at the frontier of our understanding of the causes and consequences of convergent evolution.
\end{abstract}

Keywords: adaptation, comparative biology, constraint, evolutionary ecology, phylogenetic ecology, plant-insect interactions.

\section{Introduction}

The search for convergent evolution and its causes is one way to make sense of the wonderfully bewildering biolog-

\footnotetext{
* This issue originated as the 2016 Vice Presidential Symposium presented at the annual meetings of the American Society of Naturalists.

$\dagger$ E-mail: aa337@cornell.edu.

Am. Nat. 2017. Vol. 190, pp. S1-S12. (C) 2017 by The University of Chicago. 0003-0147/2017/190S1-57341\$15.00. All rights reserved. DOI: $10.1086 / 692111$
}

ical diversity on our planet. The study of convergence is part of a program to discover repeated patterns and general principles that govern evolutionary diversification. Indeed, with the advent of non-model-omics, the study of convergent evolution is enjoying a new surge of interest, and this symposium and special issue of the American Naturalist brings together a superb group to address classic and novel questions on the topic.

Here I define convergence as the independent evolution of similar phenotypes. As has been noted many times, the intrigue of convergence was not lost on one of our greatest naturalists, Charles Darwin, when he identified traits such as luminescent organs in seemingly distantly related insects and pollen packages in distantly related plants. Darwin (1859, p. 193) wrote: "although the general appearance and function of the organ may be the same ... some fundamental difference can generally be detected ... Natural selection ... has sometimes modified in very nearly the same manner two parts in two organic beings, which owe but little of their structure in common to inheritance from the same ancestor." Darwin recognized that convergent traits are not necessarily identical in all respects and that their evolution was largely independent, although not completely so, given that all organisms ultimately share a common ancestor.

Most convergent evolution falls under the umbrella of what may be considered "constrained adaptation" - evolution that is limited by the strength of natural selection, genetic architecture, and fitness costs and benefits, all of which narrow the number of possible evolutionary outcomes. Although convergence is often interpreted as evidence of both adaptation and constraint (sagaciously reviewed by Losos [2011]), the two processes are intertwined and thus can be difficult to separate. In this context, constraint has been broadly defined as "restrictions or limitations on the course or outcome of evolution" (Arnold 1992) or, more generally, the "unequal probability of outcomes in evolution" (Schwenk 1994/1995). Issues of constraint will be important in my discussion of convergent evolution, but because of the varied historical use and 
abuse of the term (Futuyma 2010), I will frequently use the term "bias" to simply refer to the pattern of unequal probability of outcome in evolution (table 1).

As Losos (2011) pointed out, repeated trait-environment associations are suggestive of convergent adaptation, and measures of natural selection and trait function can elucidate the adaptive nature of convergent traits (box 1). Nonetheless, such measures do not address the extent to which adaptation may be constrained. Many functional traits can be under selection and yet may be limited in how they can evolve. As will be discussed later in this article, a pattern of phylogenetic bias in both the degree of convergence and the underlying mechanistic basis of convergent traits is reshaping our view of constrained adaptation. The challenge of studying convergence in our era is thus interpreting patterns in natural history on phylogenies, with repeated or divergent genetic mechanisms helping to elucidate general issues in evolution. This was the focus of the American Society of Naturalists Vice Presidential Symposium in 2016.

In this introductory article, I aim to (1) introduce general issues and concepts in the study of convergence and in particular link the study of convergence to its roots in natural history, (2) provide a framework for addressing modern questions in convergence and summarize the state of the field in terms of progress and open questions, and (3) highlight themes addressed in the subsequent articles in this special issue. The current growth in studies of convergence has been fueled by increasingly sophisticated modern analytical tools of comparative biology, the ability to pinpoint genetic mechanisms of convergence using molecular biology, and the expansion of observational (as opposed to experimental) science in the past few decades. While perhaps less common, experimental approaches and novel statistical methods in the study of convergence are helping to explain patterns with process (Lenski 2017; Mahler et al. 2017). We are beginning to study the cellular and molecular mechanisms leading to convergence in distantly related or- ganisms subject to the same selection, a critical approach to understanding phylogenetic biases in evolution (Petschenka et al. 2017; Rosenblum et al. 2017).

Convergence has also been at the center of thinking about major ecological patterns and their causes. For example, Edwards et al. (2017) seek to understand the causes of deciduousness in woody plants, a highly convergent trait that defines several global biomes. In some cases, it has been hypothesized that convergence occurs at the community level, driving greater similarity of species within a community than would be predicted from their phylogenetic relatedness (Schluter 1986). In other cases, dissimilarity evolves due to the convergent evolution of community members into different niches (Gillespie 2004; Losos 2009). Identifying traits that may be involved in community assembly are critical, as such traits are the link between evolutionary history and ecological outcomes. Together, these approaches represent an exciting frontier in the study of convergent evolution whereby we are able to study the causes and consequences of repeated evolutionary change integrating from genes to communities.

\section{Natural History: The Roots of the Study of Convergent Evolution}

Natural history concerns the description of organisms (including their scientific name), their traits (any phenotype or description of their genome), location and distribution, and interactions with the biotic and abiotic environment. Although natural history serves as a foundation for biology, there continues to be controversy about the role that natural history should play in advancing knowledge in modern biology (Greene 2005). Yet clearly "reciprocal relationships among the growth of robust theory, experimentation, and accurate natural history" (Greene 2005, p. 23) are essential for advancement in our understanding of evolutionary biology. Natural history continues to be an important source of inspira-

Table 1: Glossary of terms relevant to this article

\begin{tabular}{ll}
\hline Adaptive phenotype & A trait with a current function maintained by natural selection \\
Constraint & Restrictions or limitations on the course or outcome of evolution \\
Biased convergence & Convergent evolution where the trait consistently has the same mechanistic basis \\
Contingency & Chance events that shape evolutionary trajectories \\
Convergent adaptation & Repeated independent evolution of similar phenotypes by the same agent of natural selection \\
Convergent evolution & Repeated independent evolution of similar phenotypes \\
Environment & Abiotic conditions and biotic interactions \\
Mechanism & The causal basis of a particular trait, usually several fine-grained traits that underlie a higher-level \\
(coarse-grained) trait & Convergent evolution where the trait consistently has the same mechanistic basis within a clade \\
Phylogenetically conserved & Convergent evolution where the trait has distinct mechanistic bases, even within a clade \\
convergence & Unbiased convergence
\end{tabular}


tion, is critical for knowing and conserving what we have on the planet, and, most germane tothis article, is central to the conceptual advancement of evolutionary biology in the broadest sense. Using his classic approach of natural history and phylogenetic thinking, Greene (2017) addresses convergence in animal behavior, with implications for understanding our human selves as animals.

Natural history in the context of comparative biology has historically been a critical hypothesis generator, which has often yielded general patterns and sometimes pushed the envelope of theory - especially in our understanding of convergent evolution. Two examples from my own corner of biology, plant-herbivore interactions, illustrate this point.

By the early 1960s, the birth of chemical ecology as a discipline yielded possible explanations for chemical diversity in plants and hypothesized consequences for major patterns in biological diversification (Fraenkel 1959; Ehrlich and Raven 1964). Key hypotheses about the function of plant chemistry were informed by careful natural history observations on distantly related groups of organisms (Fraenkel 1959). In a classic study on the "raison d'être of catnip," Thomas Eisner (1964) reasoned that the chemical components (including the terpene nepetalactone) causing catnip's effects, feline euphoria, were a defense of plants against herbivorous insects. His logic followed not from the incidental effects on cats but from the fact that nearly identical compounds were produced convergently by several insects, were insecticidal, and in some cases were ejected by the animals in response to risk of predation. Eisner was an observer who poked around and followed his nose. The ejection of nepetalactone by molested beetles suggested an explanation for the function of this compound in plants.

Since then, many other defensive compounds have been found to be produced by both animals and plants, including alkaloids and cyanides. The genetic basis of such convergent evolution in defenses continues to be revealed (Jensen et al. 2011; Denoeud et al. 2014), and the hypothesis generation has not stopped with identifying the functional or mechanistic basis of these defensive traits. Conceptual developments starting in the 1970s and advancing with phylogenetic thinking since 2000 have radically improved our understanding of which plant traits repeatedly evolve together to produce multivariate strategies of defense (Feeny 1976; Kursar and Coley 2003; Agrawal and Fishbein 2006; Fine et al. 2006; Mooney et al. 2010; Johnson et al. 2014; Mason et al. 2016). In each of these studies, either closely related species (many of which inhabit different habitats) or coexisting species (which span a broad swath of phylogenetic diversity) have been studied and arrayed along multiple axes of growth and defense. A clustering of phenotypes has suggested repeatedly evolving syndromes that can be tested for their ecological effects and associations with particular environments.
Another, perhaps more grandiose academic pursuit employing convergence and natural history involved the search for general patterns or rules that might govern nature. Alfred Russel Wallace (and other contemporaries, including J. W. Slater and E. Haase) posed hypotheses about bright coloration, toxicity, and the acquisition of noxious substances from the host plants of butterfly caterpillars. Each of these naturalists, through their travels, observations, and records, noted a set of associations that lead to a hypothesis: brightly colored lepidopteran larvae obtain toxins from their host plants and use them in defense, typically against vertebrate predators. Subsequently, in the 1950s there was a race to demonstrate such sequestration of toxins by caterpillars, which, once successful, gave rise to the search for general rules of sequestration, aposematism, and mimicry (Reichstein et al. 1968). The monarch butterfly in particular was the first species shown to sequester toxins from its host plant and became a model to address such general rules (Agrawal 2017).

In a series of studies led by Miriam Rothschild between 1967 and 1973, the association between aposematism and sequestration was cemented by studies of several distantly related insect groups that feed on related host plants (von Euw et al. 1967, 1971; Rothschild et al. 1970, 1973), ultimately leading her to study 23 aposematic insect species from six insect orders that sequestered the same toxic compounds from their host plants in the Apocynaceae. Such findings opened the door to more rigorous statistical analyses, conceptual development, and theory on defense, sequestration, aposematism, and mimicry (Ruxton et al. 2004). Although the frequent association of sequestration, aposematism, and mimicry now seems obvious, it was the reciprocal interaction between natural history observations, studies of convergence, hypothesis testing, and theory development that led to the paradigm. Contemporary work addresses the genetic basis of convergent associations such as specialization and sequestration. In at least some cases, a diverse set of distantly related organisms utilizes the same mechanism to achieve adaptation to the same environmental challenge (Dobler et al. 2012; Bramer et al. 2015; Petschenka and Agrawal 2015).

\section{Mechanisms of Convergence: A New Road to Assessing the Role of Constraint?}

Traits are hierarchical in nature, from complex phenotypes (sometimes referred to as coarse-grained traits) down to the products of gene expression (i.e., fine-grained traits, beginning with messenger RNA). Higher-level traits in the hierarchy are typically dependent on several traits at lower levels (Conner and Hartl 2004), and the interpretation of convergent evolution has different meanings at these different hierarchical scales (Currie 2013). At the highest scale (or coarse grain), an ecological outcome of several traits may 


\section{Box 1: A key for categorizing, interpreting, and advancing the study of convergent evolution}

1. Has the trait repeatedly evolved?

(yes) - the trait exhibits convergent evolution; go to 2 to address why.

(no) - the trait is not convergent, and it may be difficult to assess the general importance of the trait except from logic (e.g., photosynthesis is clearly important) or functional studies. The lack of repeated evolution could potentially be addressed experimentally to understand constraints.

2. Is there a pattern of repeated trait-environment association?

(yes) - the pattern of convergent evolution is likely due to natural selection; go to $2 \mathrm{~b}$ (to understand the agents of selection) or 3 (to address biases in convergence).

(no) - the trait appears to have evolved in different environmental contexts (not a convergent adaptation), suggesting alternate functions. Go to $2 b$, which may help refine your hypothesized selective environment if you are convinced that the trait might be a convergent adaptation.

2b. Do functional analyses or other evidence suggest that the convergently evolved trait is the target of repeated natural selection by the same agent?

(yes) - the pattern of convergent adaptation is suggested; go to 3 to address selective mechanisms underlying convergence.

(no) - the trait may be correlated with traits under selection but may not be directly subject to natural selection (i.e., pleiotropy or hitchhiking).

3. Is the mechanistic basis of the convergently evolved trait identical (or nearly so) in the independent origins of the trait?

(yes) - biased convergence is suggested: there were very few possibilities for the way in which this trait could evolve; there is a single optimal solution or alternative solutions either are not as beneficial or occur with some delay and thus are less frequently realized. Go to $3 \mathrm{~b}$.

(no) - multiple mechanisms of convergence, suggesting that natural selection has favored particular phenotypic space, and the outcome was achieved in distinct ways. Go to 4 .

3b. Ask question 3 again if you can go down a mechanistic level. For example, if anthocyanins repeatedly evolved as a sunscreen, did the same genes evolve? Or if the same gene was involved in convergent evolution, was it the same specific site mutation?

4. Are the alternate mechanisms of convergent evolution phylogenetically conserved?

(yes) - phylogenetically conserved convergence: history (i.e., phylogenetic signal) and selection are both important, and there is some predictability in what traits evolve by which mechanism in different lineages. Continue to $4 \mathrm{~b}$ to understand why.

(no) - unbiased convergence: selection has resulted in a limited set of phenotypic solutions across lineages, but the means to which we get there have been mechanistically diverse even within lineages.

4b. What is the basis of the phylogenetically conserved convergence in a trait? Does a particular phenotype, gene, genetic architecture (including duplication), or extrinsic factor (such as ecology or life-history strategy) predispose a lineage to evolve the same mechanistic basis for a convergent trait? Answering this question allows us to synthesize the joint role constraint and selection in adaptation.

be the trait (or extended phenotype) that exhibits convergent evolution. For example, flight has evolved at least four times, in insects, dinosaurs, birds, and mammals. Additionally, the highly similar ecological niches employed by placental versus marsupial mammals on different continents also exemplifies convergent evolution of coarse-grained traits. If we zoom in, however, the physiological, morphological, and behavioral mechanisms that underlie convergent niches in these radiations may or may not be the same (Luo 2007).

Another such coarse-grained trait is the phenomenon of indirect defense, where protection of an organism from enemies is achieved through the attraction of, or patrolling by, animal bodyguards. The specific traits and conditions underlying indirect defense may be manifold, and these occur across diverse species and systems (e.g., ant-plants [Heil 2008], phloem-sucking hemipteran bugs [ant-tending; Styrsky and Eubanks 2007], leaf-chewing caterpillars [also ant-tending; Pierce et al. 2002], and even some fish and other vertebrates [Poulin and Grutter 1996]). The conver- gent evolution of such indirect defense as a high-level trait involves many distinct fine-grained traits, from housing structures and food rewards to volatile attractants, traps, and undiscovered mechanisms. Given that indirect defense is composed of multiple traits, we can begin to move down in scale to examine specific traits that generate indirect defense. In plants, a mechanism of indirect defense (one step lower in the hierarchy) may be extrafloral nectaries. We might next examine the chemical composition of extrafloral nectar secreted or, more mechanistically, the genes that code for the nectary and its chemical constituents. These finer-grained traits are mechanisms that generate coarse-grained traits.

Understanding underlying mechanisms is key to determining the causes of convergent evolution. For example, if convergent coarse-grained traits have distinct mechanisms, especially within a lineage, we may conclude that the evolution of those traits was less constrained. In other words, even within the backdrop of conserved genetic architecture, traits, and ecology common to a clade, the same 
evolutionary outcome was achieved by distinct means. Conversely, if mechanisms of convergence are themselves phylogenetically conserved (the mechanisms are shared among close relatives that have independently evolved the trait), then convergence likely reflects constraint. I emphasize that I am using the term "constraint" here to simply indicate bias in the outcome of evolution, here driven by shared traits in a lineage (table 1 ; box 1 ).

\section{Phylogenetic Bias in the Mechanisms of Convergence}

Several examples illustrate phylogenetic bias in the mechanisms of convergent traits. $C_{4}$ photosynthesis in plants evolved over 60 times, and several different physiological means have been employed as carbon dioxide-concentrating mechanisms in $\mathrm{C}_{4}$ plants (Sage et al. 2011). Recent evidence demonstrates the convergent recruitment of particular genes within plant lineages, but different lineages employ different genes in controlling this photosynthetic pathway (Christin et al. 2015). Also consider red coloration in flowers, a highly convergent trait that is underpinned by distinct genetic mechanisms. Evolutionary transitions to red flowers have repeatedly evolved in several lineages, but the prevalence of different mechanisms of red pigment production differs among lineages (Ng and Smith 2016). In addition to these two plant examples, resistance of animals (insects, amphibians, reptiles, and mammals) to toxic cardiac glycosides is highly convergent, and although many of the specific genetic changes are conserved across lineages, there is again some phylogenetic bias (Price et al. 1990; Croyle et al. 1997; Dobler et al. 2012; Ujvari et al. 2015).

The mechanisms by which vertebrates adapt to highelevation environments, typically involving the evolution of altered oxygenation properties of hemoglobin, have been widely studied (Storz and Moriyama 2008) and represent another case of phylogenetically conserved mechanisms in convergent evolution. In a remarkable recent study, Natarajan et al. (2016) studied 28 phylogenetic pairs of bird species and found that the oxygen affinity of hemoglobin in highland bird species was consistently higher than in closely related lowland species. Although the altered genes involved were consistent, the specific amino acid sites of substitution substantially varied. Thus, evolution was convergent at the level of individual genes but not within genes. Within hummingbirds, which had multiple origins of the highland habit, the genetic substitutions were nearly identical. Moreover, Natarajan et al. (2016) demonstrated that those genetic substitutions were functional only in the hummingbird's genetic background, not in that of more basal species. Thus, phylogenetic bias in the mechanisms of convergent evolution may be driven by the genetic background of a clade.

Phylogenetic bias in the mechanisms of convergence appears to be a general result, one that should be quantita- tively evaluated in the coming decades. Understanding this bias and its causes (such as a constraining effect of conserved genetic architecture) will lead to a greater understanding of convergence across scales. A potential exception to the pattern of phylogenetic bias in the mechanisms of convergence may be loss of functions, which can presumably occur through many mechanisms (i.e., mutations) that have the same ultimate consequence (Manceau et al. 2010; Smith et al. 2013). Thus, when the mechanisms of convergence are phylogenetically conserved (i.e., a given mechanism repeatedly evolves within a lineage but distinct mechanisms evolve between lineages), a biasing effect of genetic architecture, life history, or some other attribute of the lineage is suggested.

Biases in convergent evolution can also be studied experimentally. Comparing what has evolved in the natural world with experimental populations has the potential to reveal the extent of constraint (Weinreich et al. 2006; Stern 2013; van Ditmarsch et al. 2013). For example, both mutagenesis screens for particular phenotypes and experimental evolution studies can reveal a greater number of possibilities than exist in nature, while their natural counterparts typically reveal biased outcomes. A next step will be to reveal why certain possible mechanisms are not realized. The biasing effect of genetic architecture is the tip of the iceberg (Natarajan et al. 2016).

In some cases, advantageous mutations to groups of genes involved in adaptation may be quite limited, even in highly controlled and benign laboratory conditions. In these cases, convergence may simply be the product of constrained possibilities. However, in mutagenesis studies of microbes, plants, and animals (mostly insects), screens have typically revealed many more potentially functional mutations than those that are realized through the natural evolutionary process (reviewed in Stern 2013). For example, in the case of molecular adaptations of animals to toxic cardiac glycosides (produced by plants and some animal prey), there are relatively few mutations that have repeatedly evolved (across many orders of insects and in some vertebrates) in the target site (e.g., the ubiquitous animal enzyme or the sodium-potassium ATPase; Dobler et al. 2012, 2015; Ujvari et al. 2015). Nonetheless, animal cell mutagenesis studies reveal a greater possibility of potentially functional mutations in sodium-potassium ATPase, though this may not be the case when examined in the same genetic background (Price et al. 1990; Croyle et al. 1997). Work is just now beginning where uncommon or unrealized (but functional) mutations are being introduced to organisms (or cell lines) to address why such mutations are not typically found in nature overall or at least in particular lineages. Presumably, the uncommon or unrealized mutations do not exist because of negative fitness consequences due to either pleiotropic effects on some other function or epistatic effects given the genetic background. 


\section{Contingency, Convergence, and Parallel Evolution: New Takes on Classic Questions}

Contingency and convergence are sometimes thought to play mutually exclusive roles in evolutionary biology. In Gould's influential book, Wonderful Life (1990), he made the case for the importance of contingent or chance events that have had a profound effect on the diversification of life. Yet despite being a hero in Gould's story, Simon ConwayMorris subsequently took issue with several of Gould's theses. In fact, in two subsequent books, Conway-Morris provided many examples of convergent evolution that he argued provided evidence against Gould's conclusion of the importance of contingency (Conway-Morris 2003, 2015). Although it might be an attractive proposition that contingency and convergence are alternatives, is that truly the case? No. Convergence can be found at many levels, and yet contingent events also shape patterns of life on Earth. The polarization of the contingency and convergence debate has taken hold conceptually but is largely a false dichotomy.

Gould's contingency was conceptualized at a deep scale of chance events that shape the future (e.g., extinction of major lineages such as the dinosaurs). If certain traits evolved only once and those lineages were subject to a contingent catastrophe, then clearly contingency rules. But the importance of such contingent events is not under debate. Rather, what is at issue is whether convergence overrides the importance of these chance events, because ultimately organismal traits would return to the convergent state. In all likelihood, the truth lies somewhere in between. While chance events can reset or change the course of evolutionary history, many traits have and will continue to exhibit convergent evolution. Contingent events create evolutionary opportunities for convergent and divergent evolution. The geographic isolation of large groups of placental and marsupial mammals allowed for remarkable convergence as these groups diversified. Alternatively, certain other contingencies, such as mass extinction events, have surely shaped the diversity of life on Earth that we see today.

Experimental evolution has allowed a reconciliation of issues and deep insights into the debate over parallel, convergent, and contingent evolution (Elena and Lenski 2003). For example, when a single clone is used to found replicate populations, one can quantify whether parallel or convergent evolution occurs (Arendt and Reznick 2008). Following Stayton (2015), here I refer to parallel evolution as two lineages starting with the same character state and ultimately evolving into a different but shared character state. Many traits in Lenski's replicated long-term evolution experiment (LTEE; Lenski 2017) have repeatedly evolved (typically in parallel; e.g., Meyer et al. 2010; Lenski et al. 2015). Alternatively, other traits have evolved only in single populations (Blount et al. 2008). Experimental evolution approaches have allowed us to ask, When there is a lack of convergence, does this indicate that contingency dominates? In the LTEE, only one out of 12 replicate populations evolved the ability to utilize citrate as a carbon source, and this emerged after 30,000 generations. Interestingly, the citrate-utilizing line shows repeatable evolution of citrate use after 20,000 generations. This has been tested by restarting the experiment at different time points using frozen ancestors. The genetic architecture was potentiated by specific mutations after 20,000 generations, and these mutations serve to repeatedly favor subsequent mutations that allow for the use of citrate (Blount et al. 2012)but in only one out of 12 populations that started from the same clone. In an observational and phylogenetic context, similar patterns have been observed, where a starting character state has a strong influence on the subsequent macroevolutionary trajectory taken (Smith et al. 2013).

Various authors have proposed that the repeated evolution of traits may be potentiated by past events. For example, an original trait may have a low probability of evolving (i.e., dependent on contingencies, as in the case of citrate use described above), yet what follows is highly repeatable. Interestingly, there is no agreed-upon terminology for such traits that then cause a bias, predisposition, or inherency (Conway-Morris 2003) toward a particular evolutionary outcome. Traits that enable (Donoghue 2005), channel (Gould 2002), and potentiate (Blount et al. 2008) or that are precursors (Marazzi et al. 2012) to subsequent change have all been suggested. The extent of convergence and shared mechanisms of the traits that convergently evolve may frequently depend on such phenomena.

In experimentally tractable systems, if one has a hypothesis for what causes the predisposition, this can be addressed by introducing specific changes while controlling for other aspects of genetic architecture. In some cases, a particular gene may cause the predisposition (i.e., epistasis), and in other cases it may be a constellation of genetic factors (including gene duplication; Riehle et al. 2001; Stern 2013). Even the ecology or life history of a group may cause these events, and such predispositions may well be evident in phylogenies (Marazzi et al. 2012). In either case, a somewhat infrequent event may predispose a lineage to convergently evolve subsequent adaptations. As discussed above and in box 1 , the same mechanism may repeatedly (convergently) evolve within lineages, but different mechanisms may be convergently employed in other lineages, all toward the same end of adapting to a particular selective agent. The examples of phylogenetically conserved convergences discussed above $\left(\mathrm{C}_{4}\right.$ photosynthesis, red flower color in plants, animal adaptation to high elevation, and animal resistance to cardiac glycosides; Dobler et al. 2012; Christin et al. 2015; Ujvari et al. 2015; Natarajan et al. 2016; Ng and Smith 2016) are outstanding candidates to study the joint role of biases and selection in adaptation. 


\section{What Is the Expected Level of Convergence?}

Convergent evolution is inherently intriguing because at some level it seems unexpected, especially when convergent traits have the same genetic underpinnings. Nonetheless, where possible, the extent of convergence should be contextualized against a null model or hypothesis of what might be expected, both by chance and owing to selective processes. Stayton has argued for the importance of genetic drift in developing null models for the extent of convergence (Stayton 2008). Below I consider ecological opportunity, genetic architecture, loss versus gain of functions, and genes of small versus large effect when generating expectations for the level of convergent evolution.

In a qualitative sense, the likelihood of convergence may be driven by ecological opportunity, which may be based on what resources are in excess and can be easily employed. Viewed through this lens, it is not surprising that plants have evolved extrafloral nectaries many times (Weber and Keeler 2013). Sugar is often not limiting to plants, predacious ants often live nearby, and plant genomes share many genes. Similarly, as discussed above, many phloemsucking hemipteran bugs have evolved indirect defense by ants who drink their sugary honeydew excretions (Styrsky and Eubanks 2007). Their honeydew is a waste product, but nonetheless, there are closely related Hemiptera that are ant tended or not, and the evolution of tending typically involves specific morphological traits as well as changes in the amount or composition of honeydew (Völkl et al. 1999; Fischer et al. 2002; Shingleton and Stern 2003; Shingleton et al. 2005). Thus, the Hemiptera apparently have an ecological resource in excess, which, with some modification, may be highly sought after by an interaction partner.

In contrast, one group of leaf-chewing insects, the Lycaenid butterflies (and some close relatives) have evolved a nectar-producing gland and recruit ants as defensive bodyguards as well (Pierce et al. 2002). Within the Lepidoptera this may well be the only case of the evolution of defense by ants, and to my knowledge it has not been reported for beetles or flies. Some gall wasp lineages have repeatedly evolved the ability to induce nectar production on their galls (produced by their host plants), and they gain protection from patrolling ants (Inouye and Agrawal 2004; Nicholls et al. 2016). As far as I know, no vertebrate produces nectar to reward tending ants. Thus, among animals, the high ecological opportunity for the evolution of defense by ants appears to have widely spurred ant-hemipteran mutualism (and the traits that support it), whereas what caused the limited (single?) evolution of the same strategy in Lepidoptera is more difficult to know. Perhaps it was a chance event or circumstance in the history of the Lycaenids. The challenge for us now is to somehow quantitatively address the expectation for such convergences, perhaps depending on both extrinsic attributes such as ecological opportunity and intrinsic attributes such as genetic architecture.

Given the prevalence of epistasis, gene duplication, and effects of genetic background on the fitness advantages of particular mutations (e.g., Riehle et al. 2001; Weinreich et al. 2006; Tenaillon et al. 2012; Kryazhimskiy et al. 2014), the null expectation of convergence should be informed by knowledge of such genetic effects. Given a particular genetic background, a new mutation may be more compatible or functional and result in having higher fitness than without that specific background. As discussed above, we are at just the beginning of being able to understand biases in the extent of genetic convergence, but given that they exist, we should work toward a predictive framework that would include null expectations based on genetic architecture.

It has been suggested that loss of function mutations may on average be less mechanistically convergent than gain of functions (Arendt and Reznick 2008; Manceau et al. 2010; Smith et al. 2013). It is certainly the case that convergent loss of pigmentation, which often occurs through mutations in the same gene, occurs by distinct mutations at different sites (Protas et al. 2006). The rampant convergent loss of the gas (swim) bladder among teleost fishes also occurs by many distinct mechanisms; even losses within a single species, wild-caught zebra fishes, occurred by over 20 distinct mechanisms (McCune and Carlson 2004). In a sense, loss of function is analogous to a highly polygenic trait, since genetic modifications at many locations will result in the same phenotypic outcome.

Are convergent phenotypic traits that are controlled by many genes also less likely to have a common mechanistic basis? The genetic basis of body size is highly polygenic, and yet geographical clines (e.g., across latitude) in phenotype are highly repeatable across species and convergently evolve in native and introduced populations (e.g., see Lomolino 2005 for a review on vertebrates). Nonetheless, the genetic basis of such convergent phenotypic clines appears to occur by divergent mechanisms (reviewed for Drosophila in Gilchrist and Partridge 1999; Huey et al. 2000; Arendt and Reznick 2008). A polygenic basis for adaptation to high temperature may also underlie convergence in experimental studies of Escherichia coli. For example, experimental adaptation to high temperature in over 100 replicate lines revealed that any given pair of lineages shared relatively few nonsynonymous point mutations (2.6\%), but at a higher hierarchical level, modified genes and operons were much more likely to be shared ( $>20 \%$; Tenaillon et al. 2012). In the adaptation of fitness in 65 closely related yeast genotypes, Kryazhimskiy et al. (2014) demonstrated that although fitness evolved in highly repeatable trajectories, the specific mutations were highly variable and dependent on epistasis based on past fitness gains. In contrast to these studies on polygenic trait evo- 
lution, in cases of resistance to particular toxins or diseases with highly specific target sites (with genes of major effect), much stronger convergence at the molecular level has been expected and observed (ffrench-Constant et al. 1998; Ashfield et al. 2004; Dobler et al. 2012; Farhat et al. 2013; Yang et al. 2013; Brodie and Brodie 2015; Ujvari et al. 2015).

\section{Beyond Mechanism: Evolutionary and Ecological Consequences of Convergence}

Thus far, this article has focused on interpreting patterns of convergent evolution and integrating studies to understand the causes of convergence. As discussed above, the evolution of particular genes or traits may predispose a lineage to converge on a particular solution in response to natural selection. In addition, when convergence occurs (at whatever mechanistic level), are there predictable outcomes for the evolution and ecology of a lineage?

Perhaps the greatest interest among evolutionary biologists in consequences of convergence has come in the form of searching for macroevolutionary key innovations, those traits that evolve and allow organisms to interact with the environment in new ways and increase the net lineage diversification rate (Hunter 1998). Although there are now sophisticated analytical tools to detect shifts in diversification rates on phylogenies, even if they occur only once (Rabosky 2014), single occurrences have little ability to provide generality in terms of how traits impact diversification. Even though we have little understanding of why particular traits may facilitate speciation (or retard extinction; Heard and Hauser 1995; Futuyma and Agrawal 2009), recent work on plant-insect interactions suggests that such traits exist (Fine et al. 2004; Kaminski et al. 2010; Forister et al. 2011).

Indeed, our best cases of traits acting as key innovations, especially from traits that have repeatedly evolved, come from the defensive traits of plants: latex (Farrell et al. 1991) and extrafloral nectaries (Weber and Agrawal 2014). In both cases, the traits have convergently evolved in numerous plant families, the traits are associated with defense against herbivores, and lineages that have evolved these traits have higher diversification rates than closely related lineages lacking the traits. Convergent evolution of these defensive traits has had profound and predictable consequences for plant diversification. Remarkably, on the coevolutionary flip side, herbivory as a trait in insects is also an iconic case of a convergent trait (feeding strategy) that is widely associated with elevated diversification rates (Mitter et al. 1988; Wiens et al. 2015). Future work will certainly identify other convergent key innovations, but more importantly, we must address how and why the traits impact speciation or extinction.
From an ecological perspective, there is tremendous interest in understanding the species and traits that may cause predictable outcomes in community assembly and composition. Such processes may occur over long periods of time (which include evolutionary change) or over shorter periods of time (e.g., following disturbance or during succession). Do particular species and their traits, once present in a community, fundamentally change the course of assembly? In the classical cases of adaptive radiations in bounded communities (i.e., lakes and islands), there is often an association between convergently evolved animal ecomorphs and assembly of a fauna of related species (Losos et al. 1998; Gillespie 2004; Turner 2007). Nonetheless, cause and effect between the convergent traits and assembly of the community can be difficult to discern. The consideration of traits that modulate positive and negative species interactions is an especially important frontier, as both can impact community structure, though in different ways. Fukami et al. (2017) take on the issue of convergence in plant mycorrhizal associations and how these may have shaped predictable outcomes of community structure in diverse tropical forests.

In a general sense, convergent traits may strongly impact the process of community assembly through a few different processes. One metric of community assembly is the phylogenetic structure of a community, defined as a nonrandom pattern of evolutionary relatedness among species (Kraft et al. 2007). In the simplest case where habitat filtering is critical, convergence in traits among species will result in a pattern of even (sometimes called over-dispersed) communities, those where species are less closely related than would be expected by chance (Cavender-Bares et al. 2004). Conversely, if traits are phylogenetically conserved (e.g., tolerance of some stress), habitat filtering will result in phylogenetically clustered communities. However, when traits are evolutionarily conserved and yet there is selection for dissimilarity within a community (because of competition or other negative species interactions among close relatives), the outcome will result in an even community. Finally, for the case where some habitat filtering occurs as well as where species interactions are important and species' traits are convergent (likely the most typical scenario), the community outcome is unclear (Kraft et al. 2007). Thus, quantitatively parsing out the impact of convergent evolution on community structure is a critical frontier in understanding the role of deep evolutionary processes in community assembly.

\section{Looking Back and Looking Forward}

There are two related issues for why convergence provides such an important and compelling approach to biology, especially when describing phenomena or taking a natural 
history approach. As pointed out by Felsenstein (1985), independent evolution provides statistical power in comparative biology. Correcting for phylogeny, though sometimes having a pejorative connotation, is simply about making robust statistical arguments about the association between two phenomena. Whether two traits show correlated evolution, a trait repeatedly evolves in a particular environment, or the repeated evolution of a trait is consistently associated with increased diversification, convergence in all cases provides power for stronger inference than if the association occurred only once. This is not to downplay the importance of contingent events that occurred only once; indeed, these may be some of the most critical events in evolutionary history. Nonetheless, to gain insight into whether there are general rules in biology, there is no substitute for evolutionary replication. Evolutionary replication allows us to come closer to understanding the cause of a particular association because, if repeated in independent lineages, the genetic background, life history, and ecology of the distinct lineages are likely to be different, and yet the association still stands.

The current renaissance in descriptive biology has grown from exploring the natural histories of the genomes of many organisms (e.g., Parker et al. 2013), engaging in new forms of discovery, and comparative analyses. There has been tremendous growth in the analysis of ecological gradients and understanding the natural pattern of species traits, distributions, and interactions along latitude, altitude, and other gradients. Part of this revolution has come from a renewed interest in trait-based ecology, the availability of phylogenetic information, and climatic databases. Given this resurgence, I conclude with four hopeful messages. First, let's embrace this interest, as natural history is an important basis of inspiration, discovery, and the conservation of species. Second, the use of convergence can provide rigor and replication to address some of the biggest questions, ranging from understanding constraints to diversification. Third, there is tremendous potential to understand the underlying drivers of convergence by addressing the extent to which the mechanisms of convergent traits are biased by the phylogenetic lineage in which they evolve (box 1). Last, the combined use of comparative biology and experimentation (ranging from mutagenesis screens to reciprocal transplant experiments) will be critical in advancing biology (Weber and Agrawal 2012).

\section{Acknowledgments}

Funding and stimulation to think about this topic was provided by the John Templeton Foundation and especially P. Wason. Funding for this symposium and the speakers was provided by the American Society of Naturalists. For discussion, I am grateful to L. Arcila Hernandez, K. Böröczky, J. Elias, H. Greene, A. Hastings, K. Holmes, H. Inamine,
P. Jones, M. Kirkpatrick, R. Lenski, A. McCune, K. Niklas, R. Petipas, B. Reed, and S. Scheiner. This article was improved by comments from J. Bronstein, J. Conner, J. Losos, J. Maron, A. McCune, J. Thompson, and M. Weber.

\section{Literature Cited}

Agrawal, A. A. 2017. Monarchs and milkweed. Princeton University Press, Princeton, NJ.

Agrawal, A. A., and M. Fishbein. 2006. Plant defense syndromes. Ecology 87:S132-S149.

Arendt, J., and D. Reznick. 2008. Convergence and parallelism reconsidered: what have we learned about the genetics of adaptation? Trends in Ecology and Evolution 23:26-32.

Arnold, S. J. 1992. Constraints on phenotypic evolution. American Naturalist 140(suppl.):S85-S107.

Ashfield, T., L. E. Ong, K. Nobuta, C. M. Schneider, and R. W. Innes. 2004. Convergent evolution of disease resistance gene specificity in two flowering plant families. Plant Cell 16:309-318.

Blount, Z. D., J. E. Barrick, C. J. Davidson, and R. E. Lenski. 2012. Genomic analysis of a key innovation in an experimental Escherichia coli population. Nature 489:513-518.

Blount, Z. D., C. Z. Borland, and R. E. Lenski. 2008. Historical contingency and the evolution of a key innovation in an experimental population of Escherichia coli. Proceedings of the National Academv of Sciences of the USA 105:7899-7906.

Bramer, C., S. Dobler, J. Deckert, M. Stemmer, and G. Petschenka. 2015. $\mathrm{Na}+/ \mathrm{K}+$-ATPase resistance and cardenolide sequestration: basal adaptations to host plant toxins in the milkweed bugs (Hemiptera: Lygaeidae: Lygaeinae). Proceedings of the Roval Societv B 282:20142346.

Brodie, E. D., and E. D. Brodie. 2015. Predictably convergent evolution of sodium channels in the arms race between predators and prey. Brain, Behavior, and Evolution 86:48-57.

Cavender-Bares, J., D. D. Ackerly, D. A. Baum, and F. A. Bazzaz. 2004. Phylogenetic overdispersion in Floridian oak communities. American Naturalist 163:823-843.

Christin, P.-A., M. Arakaki, C. P. Osborne, and E. J. Edwards. 2015. Genetic enablers underlying the clustered evolutionary origins of $\mathrm{C}_{4}$ photosynthesis in angiosperms. Molecular Biology and Evolution 32: 846-858.

Conner, J. K., and D. L. Hartl. 2004. A primer of ecological genetics. Sinauer, Sunderland, MA.

Conway-Morris, S. 2003. Life's solution: inevitable humans in a lonely universe. Cambridge University Press, Cambridge.

2015. The runes of evolution: how the universe became selfaware. Templeton, West Conshohocken, PA.

Croyle, M. L., A. L. Woo, and J. B. Lingrel. 1997. Extensive random mutagenesis analysis of the $\mathrm{Na}+/ \mathrm{K}+$-ATPase $\alpha$ subunit identifies known and previously unidentified amino acid residues that alter ouabain sensitivity implications for ouabain binding. European Lournal of Biochemistry 248:488-495.

Currie, A. 2013. Convergence as evidence. British Journal for the Philosophv of Science 64:763-786.

Darwin, C. 1859. On the origin of species by means of natural selection, or the preservation of favoured races in the struggle for life. Murray, London.

Denoeud, F., L. Carretero-Paulet, A. Dereeper, G. Droc, R. Guyot, M. Pietrella, C. Zheng, et al. 2014. The coffee genome provides in- 
sight into the convergent evolution of caffeine biosynthesis. Science 345:1181-1184.

Dobler, S., S. Dalla, V. Wagschal, and A. A. Agrawal. 2012. Community-wide convergent evolution in insect adaptation to toxic cardenolides by substitutions in the Na,K-ATPase. Proceedings of the National Academv of Sciences of the USA 109:13040-13045.

Dobler, S., G. Petschenka, V. Wagschal, and L. Flacht. 2015. Convergent adaptive evolution - how insects master the challenge of cardiac glycoside-containing host plants. Entomologia Experimentalis et Applicata 157:30-39.

Donoghue, M. J. 2005. Key innovations, convergence, and success: macroevolutionary lessons from plant phylogeny. Paleobiology 31 77-93.

Edwards, E. J., D. S. Chatelet, B.-C. Chen, J. Y. Ong, S. Tagane, H. Kanemitsu, K. Tagawa, et al. 2017. Convergence, consilience, and the evolution of temperate deciduous forests. American Naturalist 190(suppl.):S87-S104.

Ehrlich, P. R., and P. H. Raven. 1964. Butterflies and plants: a study in coevolution. Evolution 18:586-608.

Eisner, T. 1964. Catnip: its raison d'être. Science 146:1318-1320.

Elena, S. F., and R. E. Lenski. 2003. Evolution experiments with microorganisms: the dynamics and genetic bases of adaptation. Nature Reviews Genetics 4:457-469.

Farhat, M. R., B. J. Shapiro, K. J. Kieser, R. Sultana, K. R. Jacobson, T. C. Victor, R. M. Warren, et al. 2013. Genomic analysis identifies targets of convergent positive selection in drug-resistant $M y$ cobacterium tuberculosis. Nature Genetics 45:1183-1189.

Farrell, B. D., D. E. Dussourd, and C. Mitter. 1991. Escalation of plant defense: do latex and resin canals spur plant diversification? American Naturalist 138:881-900.

Feeny, P. P. 1976. Plant apparency and chemical defense. Pages 1-40 in J. W. Wallace and R. L. Mansell, eds. Biochemical interaction between plants and insects. Plenum, New York.

Felsenstein, J. 1985. Phylogenies and the comparative method. American Naturalist 125:1-15.

ffrench-Constant, R. H., B. Pittendrigh, A. Vaughan, and N. Anthony. 1998. Why are there so few resistance-associated mutations in insecticide target genes? Philosophical Transactions of the Roval Society B 353:1685-1693.

Fine, P. V. A., I. Mesones, and P. D. Coley. 2004. Herbivores promote habitat specialization by trees in amazonian forests. Science 305:663-665.

Fine, P. V. A., Z. J. Miller, I. Mesones, S. Irazuzta, H. M. Appel, M. H. H. Stevens, I. Saaksjarvi, et al. 2006. The growth-defense trade-off and habitat specialization by plants in Amazonian forests. Ecology 87:S150-S162.

Fischer, M. K., W. Völkl, R. Schopf, and K. H. Hoffmann. 2002. Agespecific patterns in honeydew production and honeydew composition in the aphid Metopeurum fuscoviride: implications for antattendance. Iournal of Insect Phvsiology 48:319-326.

Forister, M. L., Z. Gompert, C. C. Nice, G. W. Forister, and J. A. Fordyce. 2011. Ant association facilitates the evolution of diet breadth in a lycaenid butterfly. Proceedings of the Roval Society B 278:1539-1547.

Fraenkel, G. 1959. The raison d'etre of secondary plant substances. Science 129:1466-1470.

Fukami, T., M. Nakajima, C. Fortunel, P. V. A. Fine, C. Baraloto, S. E. Russo, and K. G. Peay. 2017. Geographical variation in community divergence: insights from tropical forest monodominance by ectomycorrhizal trees. American Naturalist 190(suppl.):S105S122.
Futuyma, D. J. 2010. Evolutionary constraint and ecological consequences. Evolution 64:1865-1884.

Futuyma, D. J., and A. A. Agrawal. 2009. Macroevolution and the biological diversity of plants and herbivores. Proceedings of the National Academv of Sciences of the USA 106:18054-18061.

Gilchrist, A. S., and L. Partridge. 1999. A comparison of the genetic basis of wing size divergence in three parallel body size clines of Drosophila melanogaster. Genetics 153:1775-1787.

Gillespie, R. 2004. Community assembly through adaptive radiation in Hawaiian spiders. Science 303:356-359.

Gould, S. J. 1990. Wonderful life: the Burgess Shale and the nature of history. Norton, New York.

. 2002. The structure of evolutionary theory. Harvard University Press, Cambridge, MA.

Greene, H. W. 2005. Organisms in nature as a central focus for biology. Trends in Ecology and Evolution 20:23-27.

2017. Evolutionary scenarios and primate natural history. American Naturalist 190(suppl.):S69-S86.

Heard, S. B., and D. L. Hauser. 1995. Key evolutionary innovations and their ecological mechanisms. Historical Biology 10:151-173.

Heil, M. 2008. Indirect defence via tritrophic interactions. New Phytologist 178:41-61.

Huey, R. B., G. W. Gilchrist, M. L. Carlson, D. Berrigan, and L. S. Serra. 2000. Rapid evolution of a geographic cline in size in an introduced fly. Science 287:308-309.

Hunter, J. P. 1998. Key innovations and the ecology of macroevolution. Trends in Ecology and Evolution 13:31-36.

Inouye, B. D., and A. A. Agrawal. 2004. Ant mutualists alter the composition and attack rate of the parasitoid community for the gall wasp Disholcaspis eldoradensis (Cynipidae). Ecological Entomology 29:692-696.

Jensen, N. B., M. Zagrobelny, K. Hjernø, C. E. Olsen, J. HoughtonLarsen, J. Borch, B. L. Møller, et al. 2011. Convergent evolution in biosynthesis of cyanogenic defence compounds in plants and insects. Nature Communications 2:273.

Johnson, M. T., A. R. Ives, J. Ahern, and J. P. Salminen. 2014. Macroevolution of plant defenses against herbivores in the evening primroses. New Phytologist 203:267-279.

Kaminski, L. A., A. V. Freitas, and P. S. Oliveira. 2010. Interaction between mutualisms: ant-tended butterflies exploit enemy-free space provided by ant-treehopper associations. American Naturalist 176:322-334.

Kraft, N. J., W. K. Cornwell, C. O. Webb, and D. D. Ackerly. 2007. Trait evolution, community assembly, and the phylogenetic structure of ecological communities. American Naturalist 170:271-283.

Kryazhimskiy, S., D. P. Rice, E. R. Jerison, and M. M. Desai. 2014 Global epistasis makes adaptation predictable despite sequencelevel stochasticity. Science 344:1519-1522.

Kursar, T. A., and P. D. Coley. 2003. Convergence in defense syndromes of young leaves in tropical rainforests. Biochemical Svstematics and Ecology 31:929-949.

Lenski, R. E. 2017. Convergence and divergence in a long-term experiment with bacteria. American Naturalist 190(suppl.):S57S68.

Lenski, R. E., M. J. Wiser, N. Ribeck, Z. D. Blount, J. R. Nahum, J. J. Morris, L. Zaman, et al. 2015. Sustained fitness gains and variability in fitness trajectories in the long-term evolution experiment with Escherichia coli. Proceedings of the Roval Society B 282: 20152292.

Lomolino, M. V. 2005. Body size evolution in insular vertebrates: generality of the island rule. Journal of Biogeography 32:1683-1699. 
Losos, J. 2009. Lizards in an evolutionary tree: ecology and adaptive radiation of anoles. University of California Press, Oakland.

Losos, J. B. 2011. Convergence, adaptation, and constraint. Evolution 65:1827-1840.

Losos, J. B., T. R. Jackman, A. Larson, K. de Queiroz, and L. RodriguezSchettino. 1998. Contingency and determinism in replicated adaptive radiations of island lizards. Science 279:2115-2118.

Luo, Z.-X. 2007. Transformation and diversification in early mammal evolution. Nature 450:1011-1019.

Mahler, D. L., M. G. Weber, C. E. Wagner, and T. Ingram. 2017. Pattern and process in the comparative study of convergent evolution. American Naturalist 190(suppl.):S13-S28.

Manceau, M., V. S. Domingues, C. R. Linnen, E. B. Rosenblum, and H. E. Hoekstra. 2010. Convergence in pigmentation at multiple levels: mutations, genes and function. Philosophical Transactions of the Roval Society B 365:2439-2450.

Marazzi, B., C. Ané, M. F. Simon, A. Delgado-Salinas, M. Luckow, and M. J. Sanderson. 2012. Locating evolutionary precursors on a phylogenetic tree. Evolution 66:3918-3930.

Mason, C. M., A. W. Bowsher, B. L. Crowell, R. M. Celoy, C. J. Tsai, and L. A. Donovan. 2016. Macroevolution of leaf defenses and secondary metabolites across the genus Helianthus. New Phvtologist 209:1720-1733.

McCune, A. R., and R. L. Carlson. 2004. Twenty ways to lose your bladder: common natural mutants in zebrafish and widespread convergence of swim bladder loss among teleost fishes. Evolution and Development 6:246-259.

Meyer, J. R., A. A. Agrawal, R. T. Quick, D. T. Dobias, D. Schneider, and R. E. Lenski. 2010. Parallel changes in host resistance to viral infection during 45,000 generations of relaxed selection. Evolution 64:3024-3034.

Mitter, C., B. Farrell, and B. Wiegmann. 1988. The phylogenetic study of adaptive zones: has phytophagy promoted insect diversification? American Naturalist 132:107-128.

Mooney, K. A., R. Halitschke, A. Kessler, and A. A. Agrawal. 2010. Evolutionary trade-offs in plants mediate the strength of trophic cascades. Science 327:1642-1644.

Natarajan, C., F. G. Hoffmann, R. E. Weber, A. Fago, C. C. Witt, and J. F. Storz. 2016. Predictable convergence in hemoglobin function has unpredictable molecular underpinnings. Science 354:336-339.

Ng, J., and S. D. Smith. 2016. Widespread flower color convergence in Solanaceae via alternate biochemical pathways. New Phvtologist 209:407-417.

Nicholls, J. A., G. Melika, G. N. Stone, and M. A. McPeek. 2016. Sweet tetra-trophic interactions: multiple evolution of nectar secretion, a defensive extended phenotype in Cynipid gall wasps. American Naturalist 189:67-77.

Parker, J., G. Tsagkogeorga, J. A. Cotton, Y. Liu, P. Provero, E. Stupka, and S. J. Rossiter. 2013. Genome-wide signatures of convergent evolution in echolocating mammals. Nature 502:228-231.

Petschenka, G., and A. A. Agrawal. 2015. Milkweed butterfly resistance to plant toxins is linked to sequestration, not coping with a toxic diet. Proceedings of the Roval Societv B 282:20151865.

Petschenka, G., V. Wagschal, M. von Tschirnhaus, A. Donath, and S. Dobler. 2017. Convergently evolved toxic secondary metabolites in plants drive the parallel molecular evolution of insect resistance. American Naturalist 190(suppl.):S29-S43.

Pierce, N. E., M. F. Braby, A. Heath, D. J. Lohman, J. Mathew, D. B. Rand, and M. A. Travassos. 2002. The ecology and evolution of ant association in the Lycaenidae (Lepidoptera). Annual Review of Entomology 47:733-771.
Poulin, R., and A. S. Grutter. 1996. Cleaning symbioses: proximate and adaptive explanations. Bioscience 46:512-517.

Price, E., D. Rice, and J. Lingrel. 1990. Structure-function studies of $\mathrm{Na}, \mathrm{K}-\mathrm{ATP}$ ase: site-directed mutagenesis of the border residues from the H1-H2 extracellular domain of the $\alpha$ subunit. Iournal of Biological Chemistry 265:6638-6641.

Protas, M. E., C. Hersey, D. Kochanek, Y. Zhou, H. Wilkens, W. R. Jeffery, L. I. Zon, et al. 2006. Genetic analysis of cavefish reveals molecular convergence in the evolution of albinism. Nature Genetics 38:107-111.

Rabosky, D. L. 2014. Automatic detection of key innovations, rate shifts, and diversity-dependence on phylogenetic trees. PLoS ONE 9:e89543.

Reichstein, T., J. Voneuw, J. A. Parsons, and M. Rothschild. 1968. Heart poisons in monarch butterfly. Science 161:861-866.

Riehle, M. M., A. F. Bennett, and A. D. Long. 2001. Genetic architecture of thermal adaptation in Escherichia coli. Proceedings of the National Academy of Sciences of the USA 98:525-530.

Rosenblum, E. B., C. E. Parent, E. T. Diepeveen, and K. Bi. 2017. Convergent phenotypic evolution despite contrasting demographic histories in the fauna of White Sands. American Naturalist 190(suppl.):S44-S56.

Rothschild, M., J. von Euw, and T. Reichstein. 1970. Cardiac glycosides in oleander aphid, Aphis nerii. Journal of Insect Physiology 16:1141-1145.

1973. Cardiac glycosides (heart poisons) in the polka-dot moth Syntomeida epilais Walk. (Ctenuchidae: Lep.) with some observations on the toxic qualities of Amata (= Syntomis) phegea (L.). Proceedings of the Roval Society B 183:227-247.

Ruxton, G. D., T. N. Sherratt, and M. P. Speed. 2004. Avoiding attack: the evolutionary ecology of crypsis, warning signals and mimicry. Oxford University Press, Oxford.

Sage, R. F., P.-A. Christin, and E. J. Edwards. 2011. The $\mathrm{C}_{4}$ plant lineages of planet Earth. Journal of Experimental Botany 62:3155-3169.

Schluter, D. 1986. Tests for similarity and convergence of finch communities. Ecology 67:1073-1085.

Schwenk, K. 1994/1995. A utilitarian approach to evolutionary constraint. Zoology 98:251-262.

Shingleton, A. W., and D. L. Stern. 2003. Molecular phylogenetic evidence for multiple gains or losses of ant mutualism within the aphid genus Chaitophorus. Molecular Phylogenetics and Evolution 26:26-35.

Shingleton, A. W., D. L. Stern, and W. A. Foster. 2005. The origin of a mutualism: a morphological trait promoting the evolution of ant-aphid mutualisms. Evolution 59:921-926.

Smith, S. D., S. Wang, and M. D. Rausher. 2013. Functional evolution of an anthocyanin pathway enzyme during a flower color transition. Molecular Biology and Evolution 30:602-612.

Stayton, C. T. 2008. Is convergence surprising? an examination of the frequency of convergence in simulated datasets. $\underline{\text { Journal of }}$ Theoretical Biology 252:1-14.

. 2015. What does convergent evolution mean? the interpretation of convergence and its implications in the search for limits to evolution. Interface Focus 5:20150039.

Stern, D. L. 2013. The genetic causes of convergent evolution. Nature Reviews Genetics 14:751-764.

Storz, J. F., and H. Moriyama. 2008. Mechanisms of hemoglobin adaptation to high altitude hypoxia. High Altitude Medicine and Biology 9:148-157.

Styrsky, J. D., and M. D. Eubanks. 2007. Ecological consequences of interactions between ants and honeydew-producing insects. Proceedings of the Roval Society B 274:151-164. 
Tenaillon, O., A. Rodríguez-Verdugo, R. L. Gaut, P. McDonald, A. F. Bennett, A. D. Long, and B. S. Gaut. 2012. The molecular diversity of adaptive convergence. Science 335:457-461.

Turner, G. F. 2007. Adaptive radiation of cichlid fish. Current Biology 17:R827-R831.

Ujvari, B., N. R. Casewell, K. Sunagar, K. Arbuckle, W. Wüster, N. Lo, D. O'Meally, et al. 2015. Widespread convergence in toxin resistance by predictable molecular evolution. Proceedings of the National Academv of Sciences of the USA 112:11911-11916.

van Ditmarsch, D., K. E. Boyle, H. Sakhtah, J. E. Oyler, C. D. Nadell, É. Déziel, L. E. Dietrich, et al. 2013. Convergent evolution of hyperswarming leads to impaired biofilm formation in pathogenic bacteria. Cell Reports 4:697-708.

Völkl, W., J. Woodring, M. Fischer, M. W. Lorenz, and K. H. Hoffmann. 1999. Ant-aphid mutualisms: the impact of honeydew production and honeydew sugar composition on ant preferences. Oecologia 118:483-491.

von Euw, J., L. Fishelson, J. A. Parsons, T. Reichstein, and M. Rothschild. 1967. Cardenolides (heart poisons) in a grasshopper feeding on milkweeds. Nature 214:35-39.

von Euw, J., T. Reichstein, and M. Rothschild. 1971. Heart poisons (cardiac glycosides) in the lygaeid bugs Caenocoris nerii and Spilostethus pandurus. Insect Biochemistry 1:373-384.
Weber, M. G., and A. A. Agrawal. 2012. Phylogeny, ecology, and the coupling of comparative and experimental approaches. Trends in Ecology and Evolution 27:394-403.

2014. Defense mutualisms enhance plant diversification. Proceedings of the National Academv of Sciences of the USA 111:16442-16447.

Weber, M. G., and K. H. Keeler. 2013. The phylogenetic distribution of extrafloral nectaries in plants. Annals of Botany 111:12511261.

Weinreich, D. M., N. F. Delaney, M. A. DePristo, and D. L. Hartl. 2006. Darwinian evolution can follow only very few mutational paths to fitter proteins. Science 312:111-114.

Wiens, J. J., R. T. Lapoint, and N. K. Whiteman. 2015. Herbivory increases diversification across insect clades. Nature Communications 6:8370.

Yang, S., J. Li, X. Zhang, Q. Zhang, J. Huang, J.-Q. Chen, D. L. Hartl, et al. 2013. Rapidly evolving $R$ genes in diverse grass species confer resistance to rice blast disease. Proceedings of the National Academv of Sciences of the USA 110:18572-18577.

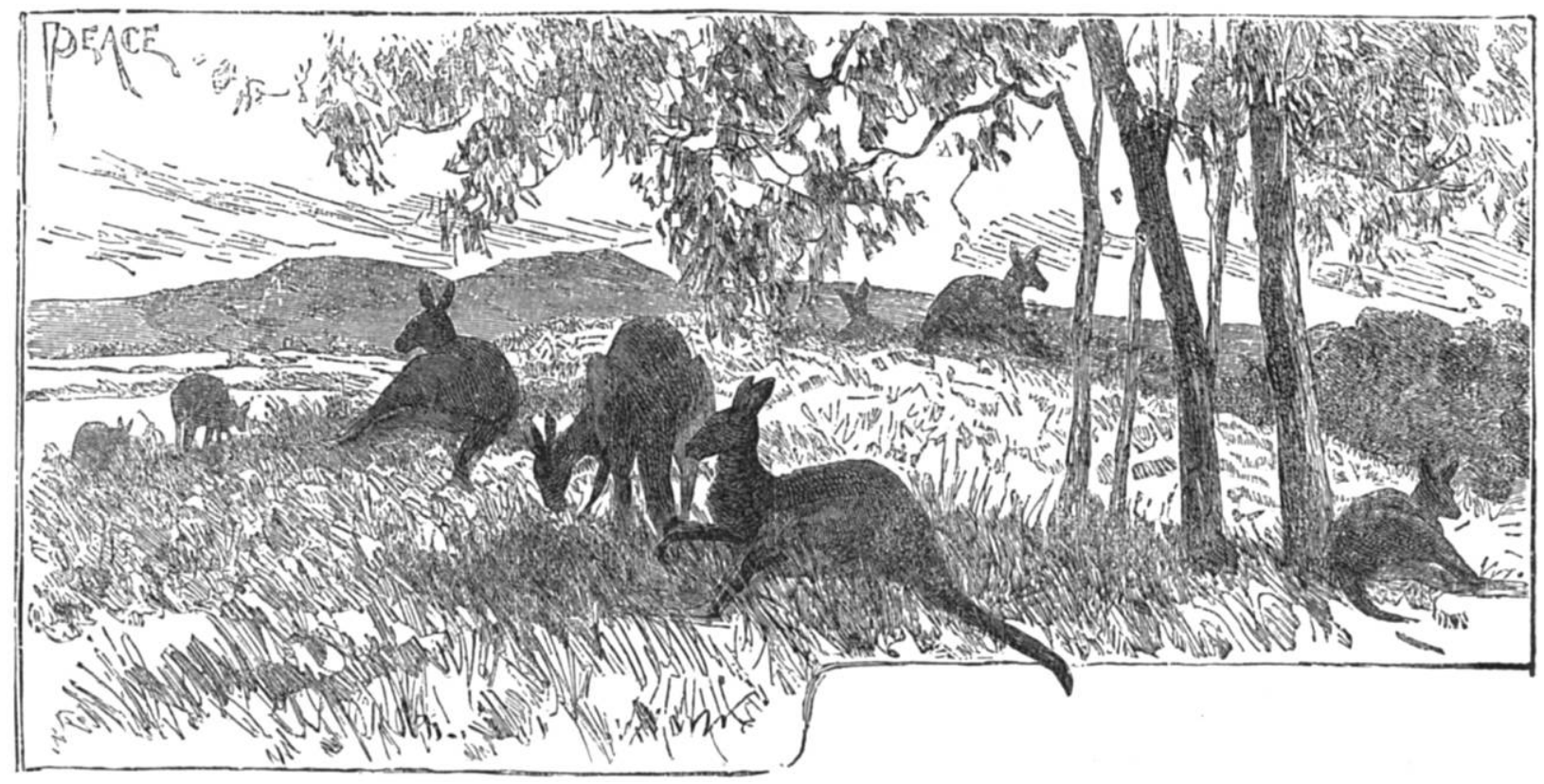

“Phases of Kangaroo Life. At Peace.” From “General Notes: Zoölogy” (The American Naturalist, 1884, 12:1271-1279). 